\title{
Analysis of the Cooperation Strategies Between HSR Express and Express Companies Based on Evolutionary Game Theory
}

\author{
Wenqin $\mathrm{CAO}^{1}$ and Xiang XU \\ School of Transportation and Logistics, East China Jiaotong University
}

\begin{abstract}
Using evolutionary game theory, the factors influencing the cooperation between high-speed railway express and express companies were further investigated. The results show that the evolutionary game path of the system is related to the excess returns, cooperation costs, excess returns, cost-sharing allocation coefficients and default amounts of both sides in the payment matrix. Specifically, when the excess returns are greater than the cost of cooperation, the two parties can cooperate, and the probability of cooperation is positively related to the excess returns and negatively related to the cost of cooperation. Penalties affect the possibility of cooperation between the two parties and are related to the parameters of cooperation.
\end{abstract}

Keywords. HSR express, evolutionary game, penalty mechanism, revenue allocation, cost apportionments

\section{Introduction}

By the end of 2019, the operating mileage of China's high-speed rail reached 35,000 $\mathrm{km}$, ranking first in the world [1]. It has achieved the ultra-high-speed development of China's high-speed rail business and accelerated the pace of cooperation between highspeed railway (HSR) express and express companies. Scholars have studied it from different perspectives. The non-cooperative game theory was applied to study the game relationship between HSR express and traditional express delivery [2]. The Stackelberg game model is used to study the optimal decision between HSR express and traditional express companies, and the option theory was introduced into the pricing model to analyze the factors influencing the strategy choice of both players [3]. The cooperative game theory approach is used to analyze the networked alliance structure and express stage market characteristics of the high-speed rail express cooperative alliance, and to explore the competition and revenue distribution problems of the high-speed rail express cooperative alliance. Based on the Nash game model and Stackelberg game model, some scholars analyze the influence of the limited rationality of cooperative entities on the cooperative strategy was considered [4]. Through evolutionary game theory, the factors that influence the cooperation between express companies and

${ }^{1}$ CAO Wenqin, School of Transportation and Logistics, East China Jiaotong University, Nanchang 330013; E-mail: bessie2310@163.com. 
railway couriers are analyzed: excess benefits and distribution coefficients, cooperation costs and sharing coefficients and the reputation of both parties. The key factors influencing the long-term and stable partnership between the railway bureau and the third-party logistics enterprise are analyzed using the evolutionary game theory and the stability of the cooperation between the two parties. In this paper, the use of evolutionary game theory, focusing on the penalty mechanism factors, excess profits and distribution coefficients, cooperation costs and apportionment coefficients and other factors.

\section{Construction of the Evolutionary Game Model}

Evolutionary game theory, based on classical game theory and evolutionary biology survival of the fittest theory [5], is effective in predicting the process of competition between groups. This paper is based on the theoretical knowledge of evolutionary game theory. The game parties are HSR express and express companies, and factors such as revenue and cost of cooperation, excess revenue and penalties are further explored, and the evolutionary path of the partnership between the two parties under the penalty mechanism is studied.

\subsection{Problem Hypothesis}

Due to the different analysis and judgment of market information and operational capacity of high-speed rail express and express companies, they cannot fully predict the outcome of the decision, in order to maximize their own interests. Therefore, both sides may take the two strategies of cooperation and non-cooperation, need to play many times, adjust and improve the strategy in order to achieve the Nash equilibrium.

First, some notations for the problem hypothesis are introduced.

- Let $x$ be the probability that HSR express will take cooperation;

- Let $y$ be the probability that express companies will take cooperation, where $x, y \in(0,1)$; When $x, y=1$, it means that both sides cooperate; when $x, y=0$, it means that both sides do not cooperate;

- $\quad$ Let $R_{1}$ and $R_{2}$ be the respective operating profits of both the HSR express and the Express company when they are not cooperating, where $R_{1}, R_{2} \geq 0$;

- Let $\Delta R$ be the excess revenue from the cooperation between HSR express and express companies when both parties cooperate, where $\Delta R \geq 0$;

- Let $a$ and (1- $a$ ) be the distribution ratio of the excess benefits of cooperation between HSR express and Express enterprises respectively, where $a \in(0,1)$;

- Let $C$ be the total cost of cooperation, which will be incurred by any party that prefers to cooperate. For example, when HSR express cooperates with express companies, both of them will invest in infrastructure capital and vehicle dispatching fees, where $C \geq 0$;

- Let $b$ be the share of HRL Express in the cost of cooperation and Let be the share of express companies in the cost of cooperation, where $b \in(0,1)$;

- Let $p$ be the penalty to be paid by one party to the other,where $p \geq 0$.in order to ensure the smooth development of cooperation, reduce the occurrence 
of default by either party, and contribute to a long-term and efficient cooperative relationship;

\subsection{Model Construction}

Based on the idea of the evolutionary game to build the payment matrix for high-speed rail express and courier companies [6], Table 1 shows the results of this payment matrix.

Table 1. Payment matrix for HSR express and Express companies

\begin{tabular}{|c|c|c|}
\hline $\begin{array}{l}\text { Express Companies } \\
\text { HSR Express }\end{array}$ & $\begin{array}{c}\text { Cooperation } \\
(y)\end{array}$ & $\begin{array}{c}\text { Non-cooperation } \\
(1-y)\end{array}$ \\
\hline $\begin{array}{l}\text { Cooperation }(x) \\
\text { Non-cooperation }(1-x)\end{array}$ & $\begin{array}{c}R_{1}+a \Delta R-b C, \quad R_{2}+(1-a) \Delta R-(1-b) C \\
R_{1}-p, \quad R_{2}-(1-b) C+p\end{array}$ & $\begin{array}{c}R_{1}-b C+p, R_{2}-p \\
R_{1}, R_{2}\end{array}$ \\
\hline
\end{tabular}

Therefore, the expected profit obtained by HSR express who adopts cooperative and non-cooperative strategy can be written by $E_{z c}$ and $E_{z n}$, where

$$
\begin{aligned}
& E_{z c}=y\left(R_{1}+a \Delta R-b C\right)+(1-y)\left(R_{1}-b C+p\right) \\
& =y a \Delta R+R_{1}-b C+p-y p=y a \Delta R+R_{1}-b C+(1-y) p \\
& E_{z n}=y\left(R_{1}-p\right)+(1-y) R_{1}=R_{1}-y p
\end{aligned}
$$

The average expected profit of HSR express is

$$
\overline{E_{z}}=x E_{z c}+(1-x) E_{z n}=x(y a \Delta R-b C+p)+R_{1}-y p
$$

According to evolutionary game theory [7], when a game player finds that it benefits less than other participants of the same population, it will actively copy the behavior of the high-yield player and thus dynamically change its strategy, so the strategy selection mechanism can be described by the replicator dynamics (RD) equation. Based on the idea of the biological evolution replicator dynamic, the replicator dynamic equation can be obtained as Eq. (4):

$$
U(x)=\frac{d x}{d t}=x\left(E_{z c}-\overline{E_{z}}\right)=x(1-x)(y a \Delta R-b C+p)
$$

Similarly, the expected profit obtained by Express companies who adopt cooperative and non-cooperative strategy can be written by $E_{e c}$ and $E_{e n}$. Where

$$
\begin{aligned}
E_{e c} & =x\left[R_{2}+(1-a) \Delta R-(1-b) C\right]+(1-x)\left[R_{2}-(1-b) C+p\right] \\
& =x(1-a) \Delta R+R_{2}-(1-b) C+p-x p \\
E_{e n} & =x\left(R_{2}-p\right)+(1-x) R_{2}=R_{2}-x p
\end{aligned}
$$

The average expected profit of Express companies is 


$$
\overline{E_{e}}=y E_{e c}+(1-y) E_{e n}=y[x(1-a) \Delta R-(1-b) C+p]+R_{2}-x p
$$

The replicator dynamic equation can be obtained as Eq. (8):

$$
U(y)=\frac{d y}{d t}=y\left(E_{e c}-\overline{E_{e}}\right)=y(1-y)[x(1-a) \Delta R-(1-b) C+p]
$$

\section{Model Analysis}

According to the basic idea of the Evolutionary Stable Strategy(ESS) [8], in order to get the evolutionary stable strategy, $U(x)=0$ and $U(y)=0$ should be satisfied [9], then the following five dynamic equilibrium points can be obtained:

$O(0,0) 、 A(0,1) 、 B(1,0) 、 C(1,1) 、 D\left(x^{*}, y^{*}\right)$. Where

$$
x^{*}=\frac{(1-b) C-p}{(1-a) \Delta R}, y^{*}=\frac{b C-p}{a \Delta R}
$$

According to the Malthusian equation [10], the game evolution results of the strategy selection of HSR express and Express companies in the cooperation process can be expressed as a system composed of equations (4) and (8). The Jacobi matrix of this system is shown as Figure 1

$$
\begin{gathered}
\operatorname{Det}(J)=\left|\begin{array}{cc}
\frac{\partial U(x)}{\partial x} & \frac{\partial U(x)}{\partial y} \\
\frac{\partial U(y)}{\partial x} & \frac{\partial U(y)}{\partial y}
\end{array}\right| \\
=\left|\begin{array}{cc}
(1-2 x)(y a \Delta R-b C+p) & x(1-x) a \Delta R \\
y(1-y)(1-a) \Delta R & (1-2 y)[x(1-a) \Delta R-(1-b) C+p]
\end{array}\right| \\
\operatorname{tr}(J)=(1-2 x)(y a \Delta R-b C+p)+(1-2 y)[x(1-a) \Delta R-(1-b) C+p]
\end{gathered}
$$

The Jacobi determinant (Det) of the ESS is greater than zero and the trace (Tre) is less than zero; if the trace is equal to zero, it is a saddle point [11]. Then the

\begin{tabular}{|c|c|c|c|}
\hline Equilibrium Points & $\operatorname{Det}(J)$ & $\operatorname{tr}(J)$ & Dynamic Stability \\
\hline$O(0,0)$ & + & - & ESS \\
\hline$A(0,1)$ & + & + & unstable \\
\hline$B(1,0)$ & + & + & unstable \\
\hline$C(1,1)$ & + & - & ESS \\
\hline$D\left(x^{*}, y^{*}\right)$ & - & 0 & Saddle Point \\
\hline
\end{tabular}
determinant and trace of the Jacobi Matrix for every dynamic equilibrium point can be respectively obtained. The specific analysis results are as Table 2 .

The case I: $\min \{b C,(1-b) C\}>p$, that is, this analysis considers only the liquidated damages $(p)$ less than the cost of cooperation for either party.

Table 2. Local stability analysis of the system 
According to the above propositions, that $O(0,0)$ and $C(1,1)$ are stable points for both sides of the game, respectively. The HSR and express companies evolve from unstable points $B(1,0), A(0,1)$ along saddle point $D\left(x^{*}, y^{*}\right)$ to $O(0,0)$ and along saddle point $D\left(x^{*}, y^{*}\right)$ to $C(1,1)$, respectively. Eventually, the steady state is reached at $O$ and $C$, respectively. That is, the phase diagram of the decision evolution trend is as Figure 1.

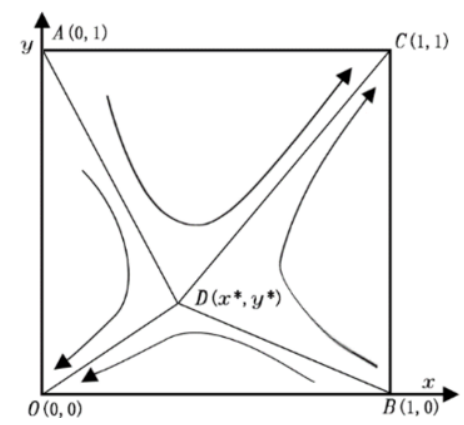

Figure 1. Phase diagram of system evolution

From the phase diagram of the system (Fig. 1), it is clear that the two strategies chosen by the system, $O(0,0)$ and $C(1,1)$, depend on the area $S_{1}$ of the geometry $(A O B D)$ and the area $S_{2}$ of the geometry (ACBD). Thus, the equation for $S_{1}$ can be obtained as Eq. (12)

$$
S_{1}=\frac{1}{2}\left[\frac{(1-b) C-p}{(1-a) \Delta R}+\frac{b C-p}{a \Delta R}\right]
$$

By analyzing Figure 1 and Eq. (12) above, the following propositions can be falsified.

The Proposition 1 The partial derivative of the function $S_{1}$ to the independent variable $x$ is as Eq. (13)

$$
\frac{\partial S_{1}}{\partial \Delta R}=-\frac{1}{2}\left[\frac{(1-b) C-p}{(1-a) \Delta R^{2}}+\frac{b C-p}{a \Delta R^{2}}\right]<0
$$

Then $S_{1}$ is a decreasing function about $\Delta R$. As the value of excess revenue $(\Delta R)$ increases, the value of $S_{1}$ decreases. That is, the system evolves towards the coordinate point $(1,1)$ destination.

The Proposition 2 The partial derivative of the function $S_{1}$ to the independent variable a is as Eq. (14)

$$
\frac{\partial S_{1}}{\partial a}=\frac{1}{2}\left[\frac{(1-b) C-p}{(1-a)^{2} \Delta R}-\frac{b C-p}{a^{2} \Delta R}\right]
$$

where $\min \{b C,(1-b) C\}>p$, Equation (14) is a continuous function for any $a$ $\in(0,1)$, and the Eqs. (15) and (16) can be obtained.

$$
\lim _{a \rightarrow 0^{+}} \frac{\partial S_{1}}{\partial a}=\lim _{a \rightarrow 0^{+}} \frac{1}{2}\left[\frac{(1-b) C-p}{(1-a)^{2} \Delta R}-\frac{b C-p}{a^{2} \Delta R}\right]=-\infty
$$




$$
\lim _{a \rightarrow 1^{-}} \frac{\partial S_{1}}{\partial a}=\lim _{a \rightarrow 1^{-}} \frac{1}{2}\left[\frac{(1-b) C-p}{(1-a)^{2} \Delta R}-\frac{b C-p}{a^{2} \Delta R}\right]=+\infty
$$

Thus, there exists $\hat{a} \in(0,1)$ such that $\frac{\partial S_{1}}{\partial \hat{a}}=0$. Similarly, the second derivative of the function $S_{1}$ to the independent variable a is as Eq. (17)

$$
\frac{\partial^{2} S_{1}}{\partial a^{2}}=\frac{1}{2}\left[\frac{(1-b) C-p}{(1-a)^{3} \Delta R}+\frac{b C-p}{a^{3} \Delta R}\right]>0
$$

Since, when $a=\hat{a}$, the first-order partial derivative of the function $S_{1}$ is equal to zero and its secondary partial conductance is constant greater than zero. Thus, there exists a value $a \in(0,1)$ such that $S_{1}$ obtains the local minimum. That is, there exists a ratio of distribution of excess profits $a$ that maximizes the possibility of cooperation between the parties to the game.

The Proposition 3 The partial derivative of the function $S_{1}$ to the independent variable $C$ is as Eq. (18)

$$
\frac{\partial S_{1}}{\partial C}=\frac{1}{2}\left[\frac{(1-b)}{(1-a) \Delta R}+\frac{b}{a \Delta R}\right]>0
$$

It's obvious to learn that $S_{1}$ is a increasing function about $C$, that is, as the cost of cooperation increases, the system evolves towards the stability point $(0,0)$.

The Proposition 4 The partial derivative of the function $S_{1}$ to the independent variable $b$ is as Eq. (19)

$$
\frac{\partial S_{1}}{\partial b}=\frac{1}{2}\left[\frac{(1-2 a) C}{(1-a) a \Delta R}\right]
$$

I: $a \in\left(0, \frac{1}{2}\right)$, that is, $\frac{\partial S_{1}}{\partial b}>0 . S_{1}$ is a increasing function about $b$ and the system evolves towards the stability point $(0,0) ; \boldsymbol{I I}: a \in\left(\frac{1}{2}, 1\right)$, that is, $\frac{\partial S_{1}}{\partial b}<0 . S_{1}$ is a decreasing function about $b$ and the system evolves towards the point $(1,1)$.

The Proposition 5 The partial derivative of the function $S_{1}$ to the independent variable $p$ is as Eq. (20)

$$
\frac{\partial S_{1}}{\partial p}=-\frac{1}{2}\left[\frac{1}{(1-a) \Delta R}+\frac{1}{a \Delta R}\right]<0
$$

$S_{1}$ is a decreasing function about $p$,that is, with increase in value of default, the system evolves towards the stability point $(1,1)$

The case II: $p>\max \{b C,(1-b) C\}$, in the case II, the only ESS point of the evolutionary process for both sides of the game is $(1,1)$. According to Table 2 , the replicated dynamic equations for the choice of strategy for HSR and express firms are as Eqs. (21) and (22)

$$
\begin{aligned}
& U(x)=\frac{d x}{d t}=x\left(E_{z c}-\overline{E_{z}}\right)=x(1-x)(y a \Delta R-b C+p) \\
& U(y)=\frac{d y}{d t}=y\left(E_{e c}-\overline{E_{e}}\right)=y(1-y)[x(1-a) \Delta R-(1-b) C+p]
\end{aligned}
$$


In Eqs. (21) and (22), when $U(x)=0$ and $U(y)=0$ should be satisfied, these five dynamic equilibrium points can be obtained: $O(0,0), A(0,1), B(1,0), C(1,1)$

Table 3. The local stability analysis of the system

\begin{tabular}{cccc}
\hline Equilibrium Points & $\boldsymbol{D e t}(\boldsymbol{J})$ & $\boldsymbol{\operatorname { t r }}(\boldsymbol{J})$ & Dynamic Stability \\
\hline$O(0,0)$ & + & + & unstable \\
$A(0,1)$ & - & unsure & Saddle Point \\
$B(1,0)$ & - & unsure & Saddle Point \\
$C(1,1)$ & + & - & ESS \\
\hline
\end{tabular}

The local stability analysis of the system is shown in Table 3. By analyzing the above results in tables, the following propositions can be obtained.

The Proposition $6 \quad C(1,1)$ is the only ESS point of the system when the case II is met, that is, the final result of evolution is that both sides choose cooperative strategy.

\section{Numerical Simulation}

This section uses numerical simulation method to further demonstrate the correctness of the above propositions and the evolution trajectory of each parameter point. The range of values in the simulation are $\Delta R \in[4000,6000], C \in[1000,3000], a \in[0.2,0.8]$ and $b \in[0.2,0.8]$. The initial values of parameters in the case I are $C=2000, \Delta R=$ $5000, b=0.4$ and $a=0.4$. As shown in Figure 2, the left vertical axis and the right axis are represented by $\Delta R, C$ ( $\Delta R$ indicates the excess revenue. $C$ indicates the total cost of cooperation) and the top horizontal axis and the bottom horizontal axis are represented by $p, S_{1}$ ( $p$ indicates the penalty. $S_{1}$ indicates the probability that both parties adopt a non-cooperative strategy).

When the default $p_{i}(i=1,2,3,4,5)$ takes different values, the value of the probability of non-cooperation $\left(S_{1}\right)$ decreases as the excess profit $(\Delta R)$ increases, while the value of $S_{1}$ increases as the total cost of cooperation $(C)$ increases. That is, the data simulation results are consistent with the propositions 1 and 3 .

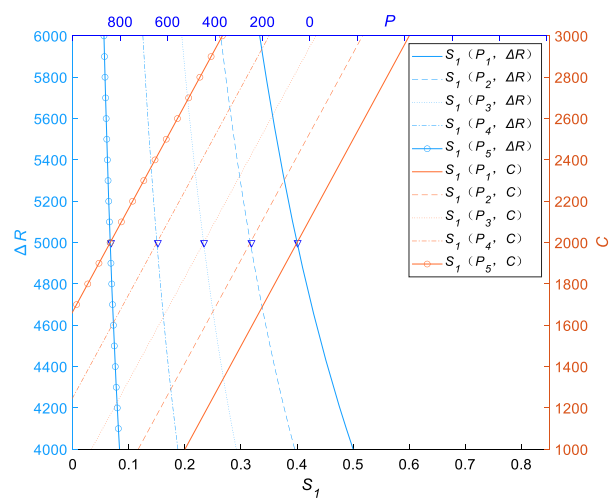

Figure 2. Dynamic evolution of parameters

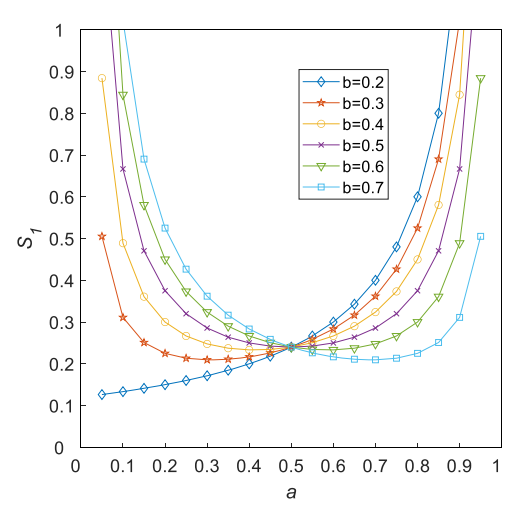

Figure 3. Dynamic evolution of parameters 
Similarly, in the case I, the value of $S_{1}$ decreases as the value of $p_{i}\left(p_{1}=0, p_{2}=200\right.$ 、 $\left.p_{3}=400 、 p_{4}=600 、 p_{5}=800\right)$ increases. Thus, the data simulation results are consistent with the proposition 5 , that is, the value of the probability of non-cooperation $\left(S_{1}\right)$ decreases as the penalty $(p)$ increases

The boundary conditions and parameters are $p=400, C=2000, \Delta R=5000, b_{1}=0.2$, $b_{2}=0.3, b_{3}=0.4, b_{4}=0.5, b_{5}=0.6, b_{6}=0.7$ and $0<a, b<1$. As shown in Figure 3 , the evolution path of the system is to quickly decrease and then rapidly increase. That is, the trajectory is a parabola with an upward opening. It is shown that there exists $a \in(0,1)$, which makes the probability of non-cooperation between the two sides of the game minimal. That is, the data simulation results are consistent with the proposition 2 .

It's satisfied the stable condition $a \in(0,0.5)$, the value $S_{1}$ increases as the value $b_{i}$ $(i=1,2 、 \ldots, 6)$ increases. Similarly, if $a \in(0.5,1)$, the value $S_{1}$ decreases as the value $b_{i}$ increases. That is, the data simulation results are consistent with the proposition 4.

\section{Conclusions and Suggestions}

The strategic choices and evolutionary trends of HSR and express companies are studied based on different parameters. The results show that if the excess revenue and the penalty are raised, it will increase the probability of adopting a cooperative strategy between gaming parties. But the total cost of cooperation is negatively related to the probability of adopting a cooperative strategy. Excess revenue allocation ratios and cooperation cost allocations also influence the choice of strategies of both parties to varying degrees.

- Increased benefits and reduced costs of cooperation. According to the analysis of the evolutionary game model. On the one hand, HSR express and Express companies need to improve management, accelerate product promotion and achieve economies of scale advantages. On the other hand, through information innovation, they should establish a strong interaction mechanism with each other and fully operate various information technologies to reduce distribution and management costs.

- Reasonable distribution of proceeds and fair allocation of costs. The two sides should establish a clear division of labor, fairness, impartiality and institutionalized cooperation mechanism, fair distribution of benefits and reasonable cost sharing, mutual trust, and actively promote win-win cooperation. Similarly, the establishment of a guarantee mechanism for information sharing can effectively reduce the risk of cooperation and ensure the fairness of profit distribution.

- Give full play to the spirit of contract and improve the penalty mechanism. In the evolutionary game model, the liquidated damages have a direct impact on the possibility of the two parties to adopt a cooperative strategy, and the reasonable liquidated damages setting can regulate and restrict the behavior of enterprises. Ultimately, it is conducive to smooth cooperation between the two sides. 


\section{Acknowledgements}

This work was supported by the Science and Technology Research Project of Jiangxi Provincial Education Department under Grant Nos. "GJJ180326".

\section{References}

[1] Wang DZ, Zhou LY, Wang Q, Yu XQ. A study on the strategy of high-speed railway express logistics center business mode selection based on evolutionary game theory. Railway Transport and Economy, 2020,42(03):24-29+35.

[2] Duan HW, Yan YS. Study on game between high-speed railway and traditional express delivery businesses based on non-cooperative game. Logistics Technology, 2015,34(07):101-105.

[3] Yang W, Zhang R. Stackelberg game model based on option pricing for crh express and traditional express. Journal of Railway Science and Engineering, 2018,15(01):39-44.

[4] Duan HW, Yan YS. Impacts of power structure on price strategy for CRH express and traditional express. Computer Integrated Manufacturing Systems, 2016,22(05):1355-1362.

[5] Maynard Smith J, G. R. Price. The Logic of Animal Conflicts. Nature, 1973(246):15-18.

[6] Xie SY. Economic Game Theory (Third Edition) Fudan University press (Shanghai), 2007:19-37.

[7] Weibull J W. Evolutionary game theory. [S. 1]: MIT Press, 1997: 45.

[8] Guo JH, Li BY, Ni M. Evolutionary game analysis of duopoly's remanufacturing entry decision. Systems Engineering-Theory\&Practice,2013,33(02):370-377.

[9] Friedman D. Evolutionary Games in Economics. Econometrica,1991, 59(3):637-666.

[10] Yu HS, Zeng A, Zhao LD. Analyzing the evolutionary stability of the vendor-managed inventory supply chains. Computers \&amp; Industrial Engineering,2008,56(1).

[11] Friedman D. on economic applications of evolutionary game theory. Journal of Evolutionary Economics, 1998, Vol.8 (1), pp.15-43. 